\title{
The difficulty with anorexia nervosa treatment: the psychological differences between anorexics' and normal eaters' approach to food
}

\begin{abstract}
On average, it takes an anorexic patient seven years to fully recover, and a majority of those patients have at least one relapse. This relapse is not due to poor self regulation, it is due to the neurological difference that is seen in anorexics that is not seen in normal eaters or bulimic patients. People with anorexia do not have a positive or rewarding neurological reaction to food, according to fMRI images, like normal eaters do. Recovering from anorexia physically takes weeks or months, but the psychological and neurobiological differences between anorexics and normal eaters, specifically their perception of and reaction to food, makes anorexia a very complex disease that can take years to fully recover from. This comprehensive paper examines the psychological differences between people with anorexia nervosa and "normal eaters" when they approach food and feeding. Anorexia patients have three broad neurocognitive inefficiencies, which include a weak central coherence, difficulties in setshifting, and impulsivity or inhibitory control, each of which contributes to the differences seen between anorexics and bulimics or normal eaters in terms of neurological functioning.
\end{abstract}

Volume I Issue $4-2015$

\author{
Becci Read \\ Human Nutrition, National College of Natural Medicine, USA \\ Correspondence: Becci Read, Human Nutrition, National \\ College of Natural Medicine, I 3 I 3 E Maple St, Suite 102 \\ Bellingham,WA 98225, USA, Email becciread7@gmail.com
}

Received: June 08, 2015 | Published: August 31, 2015

\section{Mini review}

Eating disorders have been on the rise since the first television appeared in the United States in 1928 and media began influencing what the definition of beauty was in the public eye. For many years, the development of eating disorders, especially anorexia, was contributed to negative self-esteem due to unrealistic expectations set forth by society. Recently it was discovered that neurocognitive and psychological development may predispose individuals to the development of anorexia, especially in a society where triggers for unnatural and unhealthy beauty standards are so prevalent. Anorexia nervosa is a "serious, potentially life-threatening eating disorder characterized by self-starvation and excessive weight loss", ${ }^{1}$ and has the highest rate of death of any psychological disorder, with $4 \%$ of people with anorexia dying from complications, most of these being young women between the ages of fifteen and twenty-five. ${ }^{2}$. On average, it takes an anorexic patient seven years to fully recover, and a majority of those patients have at least one relapse. This relapse is not due to poor self-regulation, it is due to the neurological difference that is seen in anorexics that is not seen in normal eaters or bulimic patients. People with anorexia do not have a positive or rewarding neurological reaction to food, according to fMRI images, like normal eaters do. Recovering from anorexia physically takes weeks or months, but the psychological and neurobiological differences between anorexics and normal eaters, specifically their perception of and reaction to food, makes anorexia a very complex disease that can take years to fully recover from.

Some professionals argue that like alcoholics, one can only be in remission from anorexia, never fully cured, if it has gotten to the point of hospitalization. This is most likely due to the neurological responses associated with food and control over food that occur in anorexia patients that do not occur in normal eaters or bulimic patients. Bulimia patients tend to have a very positive association with food itself, which is why they tend to indulge in purges. It is only later that they feel guilty about what they ate and want to correct that mistake. Anorexia patients have three broad neurocognitive inefficiencies, according to Dr. Kara Fitzpatrick, a leading psychologist in the field of eating disorders at Stanford University. These inefficiencies are not "glaringly impairing" like down's syndrome or autism; on the contrary, people with anorexia tend to have average and aboveaverage IQ levels, and are successful in school and careers. The inefficiencies that Dr. Fitzpatrick mentions are "very small areas of impaired functioning that should be concerning to us". These include a weak central coherence, difficulties in set-shifting, and impulsivity or inhibitory control, each of which contributes to the differences seen between anorexics and bulimics or normal eaters in terms of neurological functioning. ${ }^{3}$

Weak central coherence refers to how information is processed, how the "global picture" is perceived, and whether a person is highly detail oriented. It is characterized by "an overly-detailed cognitive style". ${ }^{3}$ A person with an eating disorder or who is at risk for developing an eating disorder, specifically anorexia, tend to be overly detailed, and may get "stuck" on specific details - such as weight or food portions or body image - which can impede their view and understanding of the big picture. It also impairs organization and integration of information, which can lead to a failure to grasp the global concepts and instead focus on the details of the subject. With a failed understanding of the bigger picture, the brain is not able to categorize information correctly, and the individual is not able to make connections between details and the broader concept. ${ }^{3}$. This slight neurocognitive dysfunction puts a person at risk for developing an eating disorder because they cannot see how their actions will develop into very serious consequences, they can only see their actions as a means for achieving a specific goal; in this case, a target weight or body image.

Set-shifting, or cognitive flexibility, is a very important skill that is learned during adolescence and adulthood, and has to do with the ability to move fluidly between thoughts, ideas, and concepts. People 
with eating disorders are sometimes described as "stubborn" or "inflexible", and while this is true to a certain extent, their disposition is not just them being stubborn, it is how their brain processes, stores, retrieves, and understands information that sets them apart. Anorexic individuals, or people at risk for developing anorexia, have difficulty shifting perspective, and tend to have a rigid thought process, which disables them from being willing and open to changes in their behavior and solving problems because they are unable to think beyond what they are thinking about currently. ${ }^{3}$ These problems snowball into difficulties understanding and managing ambiguity as well as planning for the future. People with anorexia like to have control, and tend to have perfectionistic tendencies due to these neurocognitive processes, which lead them to feel anxious about ambiguity and the future, so they try to take control in whatever way they know how. They are good at defining "black and white" situations or behaviors, and following through with set plans, but fluid and dynamic conditions tend to be difficult for people with this mindset. It is very trying to let go of control or change plans, which is why people with serious anorexia tend to be very successful in their goals of losing weight and sticking to a calorie restriction. This is also why it is extraordinarily difficult to change an anorexics lifestyle and eating habits in a rehabilitation setting.

Impulsivity and inhibitory control are two other skills that are learned during adolescence and early adulthood, generally between the ages of fourteen and twenty-four. People with anorexia nervosa have a greater inhibitory control. In fact, one of the contributing factors to developing anorexia is "a thinking style or using parts of the brain in such a way that it makes it easier to not do than to do". ${ }^{3}$ For instance, effortful control seems to be a baseline process that makes it easier for people with anorexia to not do something, such as indulge in dessert or fatty foods, than to engage. This is primary difference between bulimia nervosa and anorexia nervosa because bulimics have poor inhibitory control, and cannot seem to stop themselves from eating, and it is the later guilt that drives them towards their purging habits; whereas, anorexics inhibit their impulsivity before they take action. Research shows that all three of these neurocognitive impairments, but especially weak central coherence, persists even after anorexia has been treated physically through a refeeding program.

In a study conducted by Santel and colleague in Germany in 2006, fMRI images were compared between anorexia nervosa patients and a control group that had no history of disordered eating or unhealthy relationships with food. They also rated hunger and food stimuli based on self-reporting by the participants, and found that hunger led to a stronger positive rating of food in the control group than it did in the anorexia patients, meaning that even when hungry, anorexics rated food less pleasant than the controls. In a comparison of fMRI images between these two groups in which they were shown pictures of food and non-food items in both a hungry and satiated state, anorexia patients had a decreased activation in the primary taste cortex as well as the postcentral gyrus, associated with somatosensory and gustatory projections, in both a hungry and satiated state when compared to normal eaters. ${ }^{4}$ This lower activation of brain areas that control desire for food may facilitate the fasting that is associated with anorexia nervosa, and explains why refeeding and rehabilitation treatment is so challenging to initiate for patients with anorexia. These findings illustrate that food is not a pleasant experience for anorexics, and that combined with the altered neurocognitive processes found in anorexia nervosa individuals explains why treatment is so difficult for these patients to adhere to. Eating is not pleasant, they have difficulties with change, and they cannot see beyond their current thought process into the bigger picture, making it extraordinarily hard for them to embrace psychological, nutritional, and medical treatment.

Another aspect of anorexia that makes adherence to treatment more difficult is the development of the internet and social media. Anorexia is considered a lifestyle, not a disease, by both professionals specializing in eating disorders and individuals with anorexia. There is an entire online support system for people with eating disorders, and "though they don't cause eating disorders, they can perpetuate them". ${ }^{5}$ They reference anorexia as "Ana" and bulimia as "Mia". "'Ana' or 'Mia' is fondly referred to as a sufferer's female 'friend", and takes on humanistic traits on these websites. ${ }^{5}$ One of the biggest support networks is MyProAna, which is a forum where people with anorexia share strategies and techniques in order to "stay on track" with their anorexia lifestyle. These strategies range from how to trick the body into thinking it is not hungry to restricting calories without others noticing. ${ }^{6}$ The forum has a disclaimer at the top of the page that states "MPA is a forum and community to offer support and recovery for those afflicted with an eating disorder. It is not a place to encourage people to further their eating disorder. Please be cognizant of this and make MPA the support community it needs to be"; however, some of the posts on this forum include: "how long can you last on liquids alone" and "how many calories a day...". The first is full of posts saying one could live on liquids alone indefinitely, filled out with anecdotes of how long personally they have gone in the past, or currently. The second is sixty posts, each with a response stating the individual consumes somewhere between 300 to 800 calories, and demeaning comments about themselves when they eat more than their goal. Each participant has their measurements, including: height, waist, hips, high weight, current weight, and goal weight under their alias. Despite the original intention of the forum, it has become the support group for maintaining an eating disorder, and illustrates how pervasive anorexia is into the neurological functioning and perspective of the individuals who suffer from this eating disorder. To them, anorexia is the ideal lifestyle, it is a means to an end, and they have the mental structure and capacity to accomplish their goals. They are not able to see past this lifestyle or comprehend the consequences that may come from maintaining it.

Anorexia is a serious, neurological disorder that affects approximately ten million women and one million men in the United States. It is more than a medical condition that can be dealt with physically, it is a psychological disorder that presents itself in slight neurocognitive abnormalities throughout development. Thought patterns are set early in life, during childhood myelination, and a stress or trauma can affect how these neurocognitive features develop. These thought patterns and utilization of the brain can influence the development of anorexia later in life. ${ }^{4}$. Traits such as perfectionism, difficulty with flexible thinking or changes in behavior, being highly detail oriented, and advanced inhibitory control increases an individual's chance of developing anorexia. Furthermore, the approach to anorexia as a purely medical disorder, which is how the esteemed Kartini Clinic in Vancouver, WA approaches the disorder, ${ }^{7}$ is wrong because of the neurological differences seen in fMRIs, as illustrated by Santel and colleagues in 2006, and the neurocognitive differences established by Dr. Fitzpatrick in 2010. The Kartini Clinic, while still a popular rehabilitation clinic in the northwest, has not embraced current research that shows that anorexia must be considered on a neurological level; treating the physical symptoms will not cure the mental disorder. In fact, there may not be a cure, only maintenance of 
a treatment, for anorexia nervosa. The relationship with the self and food must be reshaped if treatment hopes to be successful.

Anorexia must be approached from a psychological, neurological, nutritional, and medical standpoint, with an understanding of anorexic's brain chemistry and thought processes. Their focus on detail and adherence to goals can be used to their advantage, once they have decided to change their behavior. Setting new goals and utilizing their affinity to fulfilling a task can help reform their approach to food and their body. Anorexia is a complicated and involved disorder whose neurocognitive impairments make it more difficult to treat. Respecting those neurological abnormalities and using them in a positive way could be a great reform to the current medical approach to anorexia, and help patients recover faster, and with less relapse, thus reducing the risk of morbidity.

\section{Conclusion}

Anorexia must be approached from a psychological, neurological, nutritional, and medical standpoint, with an understanding of anorexic's brain chemistry and thought processes. Their focus on detail and adherence to goals can be used to their advantage, once they have decided to change their behavior. Setting new goals and utilizing their affinity to fulfilling a task can help reform their approach to food and their body. Anorexia is a complicated and involved disorder whose neurocognitive impairments make it more difficult to treat. Respecting those neurological abnormalities and using them in a positive way could be a great reform to the current medical approach to anorexia, and help patients recover faster, and with less relapse, thus reducing the risk of morbidity.

\section{Acknowledgments}

None.

\section{Conflict of interest}

The authors declare that there is no conflict of interest.

\section{Funding}

None.

\section{References}

1. http://www.nationaleatingdisorders.org/anorexia-nervosa

2. http://www.anad.org/get-information/about-eating-disorders/eatingdisorders-statistics/

3. Fitzpatrick K. New Insights into the Neurological Basis of Eating Disorders. USA: Lucile Packard Children's Hospital/Stanford Medical Center; 2010.

4. Santel S, Baving L, Krauel K, et al. Hunger and satiety in anorexia nervosa: fMRI during cognitive processing of food pictures. Brain Research. 2006;1114(1):138-148.

5. Rainey S. 'Anorexia is a lifestyle, not a disease': An investigation into harrowing online forums promoting extreme dieting. New York, USA: National Post. 2014.

6. http://www.ampag.com.au/MPA-education-forum.htm

7. http://www.kartiniclinic.com/about/ 